\title{
Preference Comparison of AI Power Tracing Techniques for Deregulated Power Markets
}

\author{
Hussain Shareef, ${ }^{1}$ Saifunizam Abd. Khalid, ${ }^{2}$ Mohd Wazir Mustafa, ${ }^{2}$ and Azhar Khairuddin' \\ ${ }^{1}$ Faculty of Electrical Engineering and Built Environment, Universiti Kebangsaan Malaysia, 43600 Bangi, Malaysia \\ ${ }^{2}$ Faculty of Electrical Engineering, Universiti Teknologi Malaysia, 81310 Johor, Malaysia \\ Correspondence should be addressed to Hussain Shareef, shareef@eng.ukm.my
}

Received 25 May 2012; Revised 4 December 2012; Accepted 9 December 2012

Academic Editor: Thomas Mandl

Copyright (C) 2012 Hussain Shareef et al. This is an open access article distributed under the Creative Commons Attribution License, which permits unrestricted use, distribution, and reproduction in any medium, provided the original work is properly cited.

\begin{abstract}
This paper compares the two preference artificial intelligent (AI) techniques, namely, artificial neural network (ANN) and genetic algorithm optimized least square support vector machine (GA-LSSVM) approach, to allocate the real power output of individual generators to system loads. Based on solved load flow results, it first uses modified nodal equation method (MNE) to determine real power contribution from each generator to loads. Then the results of MNE method and load flow information are utilized to estimate the power transfer using AI techniques. The 25-bus equivalent system of south Malaysia is utilized as a test system to illustrate the effectiveness of the AI techniques compared to those of the MNE method. The AI methods provide the results in a faster and convenient manner with very good accuracy.
\end{abstract}

\section{Introduction}

Nowadays, the electric power industry is under deregulation in response to changes in jurisdiction, technology, market, and competition. Regardless of market environment, it is essential to know whether or not, and to what extent, each power system user contributes to the usage of particular system components. This information facilitates the restructured power system to operate economically and efficiently [1]. Moreover, it brings fair pricing and open access to all system users. Because of nonlinear nature of power flow, it is difficult to determine transmission usage accurately. Therefore, it requires using approximate models, tracing algorithms, or sensitivity indices for usage allocation. Methods based on the $Y$-bus or $Z$-bus system matrices have recently received great attention since these methods can integrate the network characteristics and circuit theories into line usage and loss allocation. The method reported in [2] is based on Kirchhoff's current law (KCL), equivalent linear circuit that reaches all lines and loads. Based on the stated assumptions, a recursive procedure was used to construct the equivalent circuit for each bus. Moreover, superposition theorem was applied to the bus's equivalent circuit starting from a bus whose injected currents were known. Another circuit concept method was proposed by Chang and $\mathrm{Lu}$ [3]. It was based on the system $Y$-bus matrix and $Z$-bus modification. Starting from the load flow solution, branch current is determined as a function of generators' injected current by using information from the bus impedance matrix. Similarly, contribution to bus voltages was computed as a function of each generator current injection by decomposing the network into different networks. Using the computed voltages and currents, the power flowing on the transmission lines were unbundled. It uses approximate formulation to calculate the unbundled loss components. This algorithm utilizes the network decomposition concept as proposed by Zobian and Ilić [4] which determines the use of transmission network by individual bilateral contracts. Teng [5] proposed a systematic method, very similar to that presented in [3], to allocate the power flow and loss for deregulated transmission systems. Using similar concept, the authors of this paper introduce a modified nodal equation (MNE) method for 
real and reactive power allocation [6] in which the load buses powers are represented as a function of the generators' current and voltage.

The tracing methods $[1,7-10]$ based on the actual power flows in the network and the proportional sharing principles were effectively used in transmission usage allocation. The methods reported in $[1,9]$ are based on tracing the current and complex power from individual power sources to system loads. Based on solved load flow, the method converts power injections and line flows into real and imaginary current injections and current flows. This method has a clear physical meaning and its results are unique. Bialek [7] proposed a novel power tracing method. However, this method requires inverting a large matrix. Wu [8] proposed a graph theory to calculate the contribution factor of individual generators to line flows and loads and the extraction factor of individual loads from line flows and generators, which is theoretically efficient. This method cannot handle loop flows and losses must be removed initially. Paper [11] was based on the concept of generator "domains," "common," and "links." The disadvantage of this method is that the share of each generator in each "common" (i.e., the set of buses supplied from the same set of generators) is assumed to be same. Furthermore, the "commons" concept can lead to problems since the topology of a "common" could radically change even in the case of slight change in power flows.

Since the meshed and nonlinear nature of power system, the applications of artificial intelligence (AI) to power system become a great potential to explore, especially in power tracing problem. Mustafa et al. [12] incorporated an artificial neural network (ANN) to reactive power allocation in deregulated power system. It uses modified nodal equation [6] results to train ANN. Similarly, research has been carried out by applying feed forward ANN for energy loss problem [13]. This method is relatively simple and easy to apply for loss allocation problem. Optimization technique also has been explored in solving the power allocation problem [14]. The authors proposed a tracing compliant that minimizes overall deviation from the postage stamp allocation. Nevertheless, the approach treats the power tracing problem as a linear constraint optimization problem. In a related work, a continuous genetic algorithm (GA) for real power tracing has been proposed in [15]. The problems of this technique are that it produces multisolution results and requires long time for computation. Paper [16] proposed a support vector machine (SVM) to estimate the contribution of individual generators to loads in power systems. The SVM gives faster results but the accuracy of the result is not promising.

Basically, support vector machine (SVM) is designed to solve the classification problem [17]. Then, it is extended for the case of nonlinear function estimation. Paper [18] uses SVM for detection of abnormalities and electricity theft by incorporating the genetic algorithm to SVM. Using similar concept, the authors of this paper also adopt the hybridization of GA and least square SVM (LS-SVM) into reactive power tracing problem [19]. The new reactive power tracing method is based on manipulation of proportional sharing method [7] and application of GA to tune the performance parameters of LS-SVM.
This paper deals mainly with investigation of two different AI techniques for real power transfer allocation and identifies most appropriate AI technique that can be used in power tracing by critically comparing the qualitative and quantitative performance of the two methods.

\section{Modified Nodal Equations Method}

The derivation, to decompose the load real powers into components contributed by specific generators, starts with basic equations of load flow. Applying Kirchhoff's current law to each node of the power network leads to the equations, which can be written in a matrix form as in (1) [6]

$$
I=Y V
$$

where $V$ is a vector of all node voltages in the system, $I$ is a vector of all node currents in the system, and $Y$ is the $Y$-bus admittance matrix.

The nodal admittance matrix of the typical power system is large and sparse, therefore it can be partitioned in a systematic way. Considering a system in which there are $G$ generator nodes that participate in selling power and remaining $L=n-G$ nodes as loads, then it is possible to rewrite (1) into its matrix form as shown in (2)

$$
\left[\begin{array}{c}
I_{G} \\
I_{L}
\end{array}\right]=\left[\begin{array}{ll}
Y_{G G} & Y_{G L} \\
Y_{L G} & Y_{L L}
\end{array}\right]\left[\begin{array}{c}
V_{G} \\
V_{L}
\end{array}\right] .
$$

Solving (2) for $I_{L}$, the load currents can be presented as a function of generators' current and load voltages as shown in (3)

$$
I_{L}=Y_{L G} Y_{G G}^{-1} I_{G}+\left(Y_{L L}-Y_{L G} Y_{G G}^{-1} Y_{G L}\right) V_{L}
$$

Then, the total real power $P_{L}$ of all loads can be expressed as shown in (4)

$$
P_{L}=\operatorname{Re}\left\{V_{L} I_{L}^{*}\right\}
$$

where (*) means conjugate, substituting (3) into (4) and solving for $P_{L}$ the relationship as shown in (5) can be found

$$
\begin{aligned}
P_{L}= & \operatorname{Re}\left\{V_{L}\left(Y_{L G} Y_{G G}^{-1}\right)^{*} I_{G}^{*}\right. \\
& \left.+V_{L}\left(\left(Y_{L L}-Y_{L G} Y_{G G}^{-1} Y_{G L}\right) V_{L}\right)^{*}\right\} \\
= & \operatorname{Re}\left\{V_{L} \sum_{i=1}^{n G} \Delta I_{L}^{* I_{G}}+V_{L}\left(\left(Y_{L L}-Y_{L G} Y_{G G}^{-1} Y_{G L}\right) V_{L}\right)^{*}\right\},
\end{aligned}
$$

where

$$
\left(Y_{L G} Y_{G G}^{-1}\right)^{*} I_{G}^{*}=\sum_{i=1}^{n G} \Delta I_{L}^{* I_{G}}
$$

and $n G$ is number of generators.

Now, in order to decompose the load voltage-dependent term further in (5), into components of generator-dependent terms, (8) derivations are used. A possible way to deduce load 
node voltages as a function of generator bus voltages is to apply superposition theorem. However, it requires replacing all load bus current injections into equivalent admittances in the circuit. Using a readily available load flow result, the equivalent shunt admittance $Y_{L j}$ of load node $j$ can be calculated using (7) as

$$
Y_{L j}=\frac{1}{V_{L j}}\left(\frac{S_{L j}}{V_{L j}}\right)^{*} .
$$

$S_{L j}$ is the load apparent power on node $j$ and $V_{L j}$ is the load bus voltage on node $j$. After adding these equivalences to the diagonal entries of $Y$-bus matrix, (1) can be rewritten as

$$
V=Y^{\prime-1} I_{G},
$$

where $Y^{\prime}$ is the modified $Y$ of (1).

Next, adopting (8) and taking into account each generator one by one, the load bus voltages contributed by all generators can be expressed as

$$
V_{L}=\sum_{i=1}^{n G} \Delta V_{L}^{* I_{G}}
$$

It is now simple mathematical manipulation to obtain the required relationship as a function of generators-dependent terms. By substituting (9) into (5), the decomposed load real powers can be expressed as

$P_{L}=\operatorname{Re}\left\{V_{L} \sum_{i=1}^{n G} \Delta I_{L}^{* I_{G}}+\sum_{i=1}^{n G} \Delta V_{L}^{* I_{G}}\left(\left(Y_{L L}-Y_{L G} Y_{G G}^{-1} Y_{G L}\right) V_{L}\right)^{*}\right\}$.

This equation shows that the real power of each load bus consists of two terms by individual generators. The first term relates directly to the generators' current and the second term corresponds to their contribution to the load voltages. With further simplification of (10), the real power contribution that load $j$ acquires from generator $i$ is as

$$
P_{L j}=\sum_{i=1}^{n G} P_{L j i}^{\Delta I_{L}}+\sum_{i=1}^{n G} P_{L j i}^{\Delta V_{L}}
$$

where $P_{L j i}^{\Delta I_{L}}$ is the current-dependent term of generator $i$ to $P_{L j}$ and $P_{L j i}^{\Delta V_{L}}$ is the voltage-dependent term of generator $i$ to $P_{L j}$.

Vector $P_{L}$ is used as a target in the training process of the proposed SVM.

\section{AI Methods Used for Real Power Allocation}

The following section describes an overview of the existing artificial intelligence power transfer allocation methods, namely, ANN method [12], and the GA-LSSVM [19].

\subsection{Function Estimation Using Radial Basis Function Artificial} Neural Network (ANN). The radial basis function (RBF) ANN was first used to design artificial neural network by
Broomhead and Lowe [20]. Radial basis function offers several advantages compared to multilayer perceptron (MLP) ANN. Firstly, it can be trained using fast two stages training algorithm without the need for time consuming non-linear optimization techniques. Secondly, the RBFN possesses the property of best approximation [21]. The network consists of three layers, namely, an input layer, a hidden layer, and an output layer. The output of the RBF ANN network simply sums the weighted basis function without using any activation function. Assuming a single neuron at the output layer, the output of the RBF network is calculated using (12) as

$$
\eta(x, w)=\sum_{k=1}^{S} w_{1 k} \phi_{k}\left(\left\|x-c_{k}\right\|_{2}\right)
$$

where $\left\|x-c_{k}\right\|_{2}$ denotes the Euclidean distance between the input vector $x$ and the center $c_{k}, \phi_{k}(\cdot)$ is a basis function, $w_{1 k}$ are the weights in the output layer, and $S$ is the number of neurons (and centers) in the hidden layer.

The output of the neuron in a hidden layer is a non-linear function of the distance. In this work, the functional form of Gaussian basis function is defined in (13) as,

$$
\phi_{k}\left(\left\|x-c_{k}\right\|_{2}\right)=e^{-\left\|x-c_{k}\right\|_{2}^{2} / \beta^{2}} .
$$

Note that the Gaussian basis function is most commonly used where the parameter $\beta$ controls the width of the RBF ANN and is commonly referred to as the spread parameter. In practice, the value of $\beta$ that is too big or too small will cause degradation in the performance of the RBFN. The centers $c_{k}$ are defined points that are assumed to perform an adequate sampling of the input space. Common practice is to select a relatively large number of input vectors as the centers to ensure an adequate input space sampling. RBF ANN performs two major functions which are training and testing. Testing is an integral part of the training process since a desired response to the network must be compared to the actual output to create an error function.

3.2. Function Estimation Using Least Squares Support Vector Machine (LS-SVM). Support vector machine (SVM) is known as a powerful methodology for solving problems in nonlinear classification, function estimation, and density estimation. Least squares support vector machine (LS-SVM) is reformulated from standard SVM [22] which lead to solving linear Karush-Kuhn-Tucker systems. In LS-SVM function estimation, the standard framework is based on a primal-dual formulation. Given $N$ data set $\left\{x_{i}, y_{i}\right\}_{i=1}^{N}$, the goal is to estimate a model of the following form:

$$
y(x)=w^{T} \varphi(x)+b+e_{i},
$$

where $x \in R^{n}, y \in R$ and $\varphi(\cdot): R^{n} \rightarrow R^{n_{h}}$ is a mapping to a high dimensional feature space. Then based on model, the following optimization problem is formulated [23]:

$$
\min _{w, b, e} J(w, e)=\frac{1}{2} w^{T} w+\gamma \frac{1}{2} \sum_{i=1}^{N} e_{i}^{2}
$$

such that $y_{i}=w^{T} \varphi\left(x_{i}\right)+b+e_{i}, i=1, \ldots, N$. 


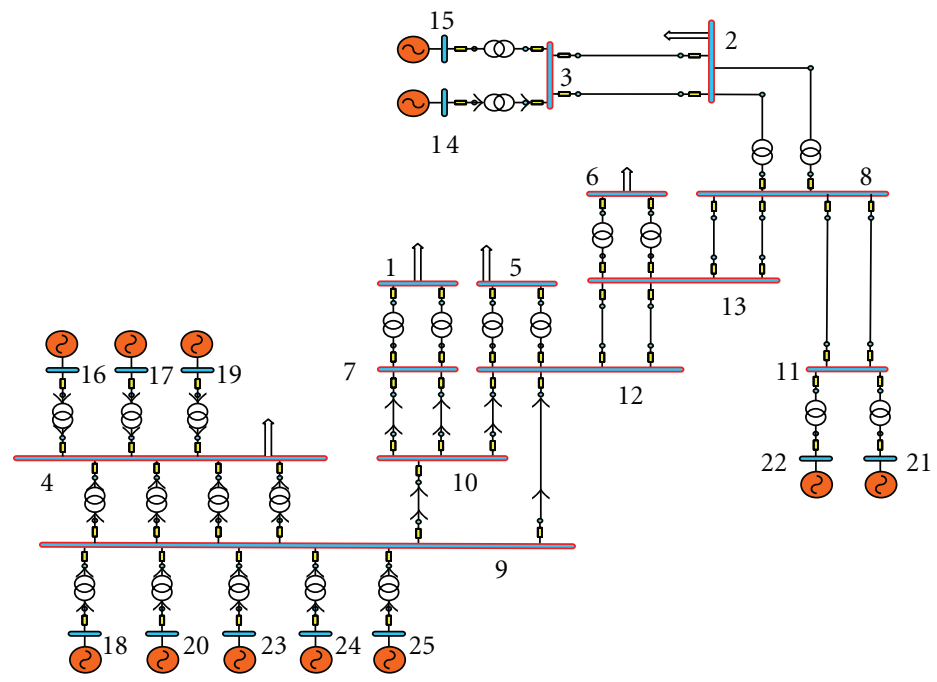

FIGURE 1: Single-line diagrams for the 25-bus equivalent practical power system.
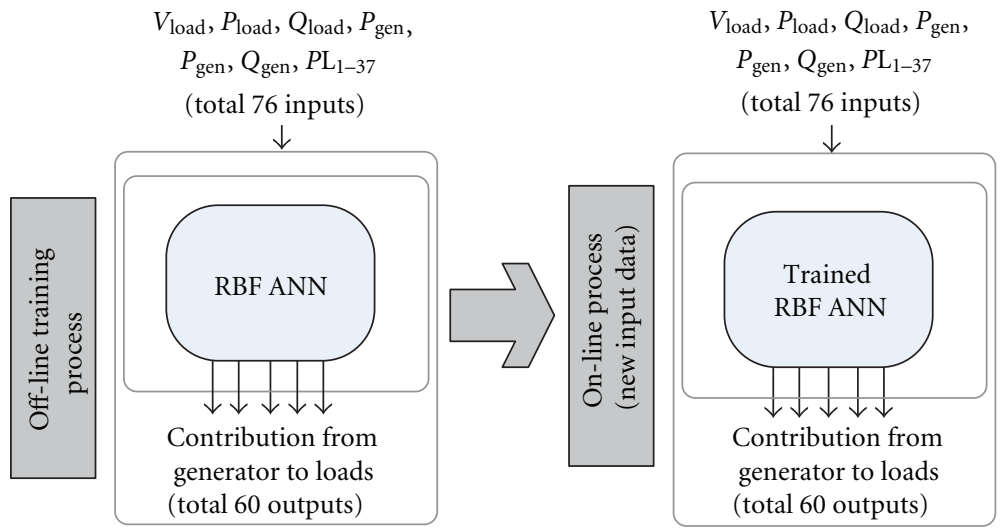

Figure 2: Description of inputs and outputs of the training and simulation data for ANN real power allocation method.

With the application of Mercer's theorem [22] for the kernel matrix $\Omega$ as $\Omega_{i j}=K\left(x_{i}, x_{j}\right)=\varphi\left(x_{i}\right)^{T} \varphi\left(x_{j}\right), i, j=$ $1, \ldots, N$, it is not required to compute explicitly the nonlinear mapping $\phi(\cdot)$ as this is done implicitly through the use of positive definite kernel functions $K$ [24].

From the following Lagrange function [23]:

$$
\begin{aligned}
\zeta(w, b, e ; \beta)= & \frac{1}{2} w^{T} w+\gamma \frac{1}{2} \sum_{i=1}^{N} e_{i}^{2} \\
& -\sum_{i=1}^{N} \beta_{i}\left(w^{T} \varphi\left(x_{i}\right)+b+e_{i}-y_{i}\right),
\end{aligned}
$$

where $\beta_{i}$ are Lagrange multipliers. Differentiating (16) with $w, b, e_{i}$, and $\beta_{i}$, the conditions for optimality can be described as follow [23]:

$$
\begin{gathered}
\frac{d \zeta}{d w}=0 \longrightarrow w=\sum_{i=1}^{N} \beta_{i} \varphi\left(x_{i}\right), \\
\frac{d \zeta}{d b}=0 \longrightarrow \sum_{i=1}^{N} \beta_{i}=0
\end{gathered}
$$

$$
\begin{gathered}
\frac{d \zeta}{d e_{i}}=0 \longrightarrow \beta_{i}=\gamma e_{i}, \quad i=1, \ldots, N, \\
\frac{d \zeta}{\beta_{i}}=0 \longrightarrow y_{i}=w^{T} \varphi\left(x_{i}\right)+b+e_{i}, \quad i=1, \ldots, N .
\end{gathered}
$$

By elimination of $w$ and $e_{i}$, the following linear system is obtained [23]:

$$
\left[\frac{0}{y} \mid \frac{1^{T}}{\Omega+\gamma^{-1} I}\right]\left[\frac{b}{\beta}\right]=\left[\begin{array}{l}
0 \\
y
\end{array}\right],
$$

with $y=\left[y_{1}, \ldots, y_{N}\right]^{T}, \beta=\left[\beta_{1}, \ldots, \beta_{N}\right]^{T}$. The resulting LSSVM model in dual space becomes

$$
y(x)=\sum_{i=1}^{N} \beta_{i} K\left(x, x_{i}\right)+b .
$$

Usually, the training of the LS-SVM model involves an optimal selection of kernel parameters and regularization 


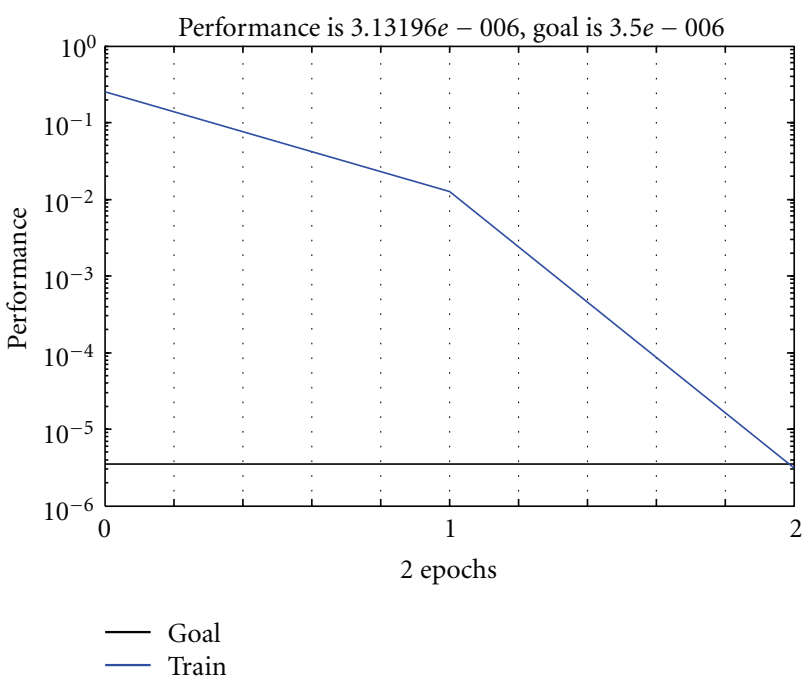

FIgURE 3: Training performance of RBF ANN.

parameter. For this paper, the RBF kernel is used which is expressed as

$$
K\left(x, x_{i}\right)=e^{-\left\|x-x_{i}\right\|^{2} / 2 \sigma^{2}} .
$$

Note that $\sigma^{2}$ is a parameter associated with RBF function which has to be tuned. There is no doubt that the efficient performance of LS-SVM model involves an optimal selection of kernel parameter, $\sigma^{2}$, and regularization parameter, $\gamma$. Thus by using GA as optimizer, an accurate result can be gained. The hybridization of GA and LS-SVM gives better accuracy and good generalization, especially in power transfer allocation problem [19].

\section{ANN Model for Real Power Allocation}

In this work, 1 RBF ANN with one hidden layer and one output layer has been chosen. The ANN power transfer allocation method is elaborated by designing an appropriate RBF ANN for the practical 25-bus equivalent power system of south Malaysia region as shown in Figure 1. This system consists of 12 generators located at buses 14 to 25 respectively. They deliver power to 5 loads, through 37 lines located at buses $1,2,4,5$, and 6 , respectively. The input samples for training are assembled using the daily load curve and performing load flow analysis for every hour of load demand. Similarly the target vector for the training is obtained from the MNE method. Input data $(D)$ for developed ANN contains variables such as load bus voltage magnitude ( $V 1$, $V 2, V 4$ to V6), real power of loads ( $P 1, P 2, P 4$ to $P 6)$, reactive power of loads ( $Q 1, Q 2, Q 4$ to $Q 6)$, real power of generators (P14 to P25), reactive power of generators (Q14 to Q25) and line real power (Pline1 to Pline37) flows, and the target/output parameter $(T)$ which is the real power transfer between generators and loads placed at buses 1, 2, 4 to 6 . Hence the networks have 60 output neurons. Figure 2 summarizes the description of inputs and outputs of the training and testing for ANN for real power allocation.

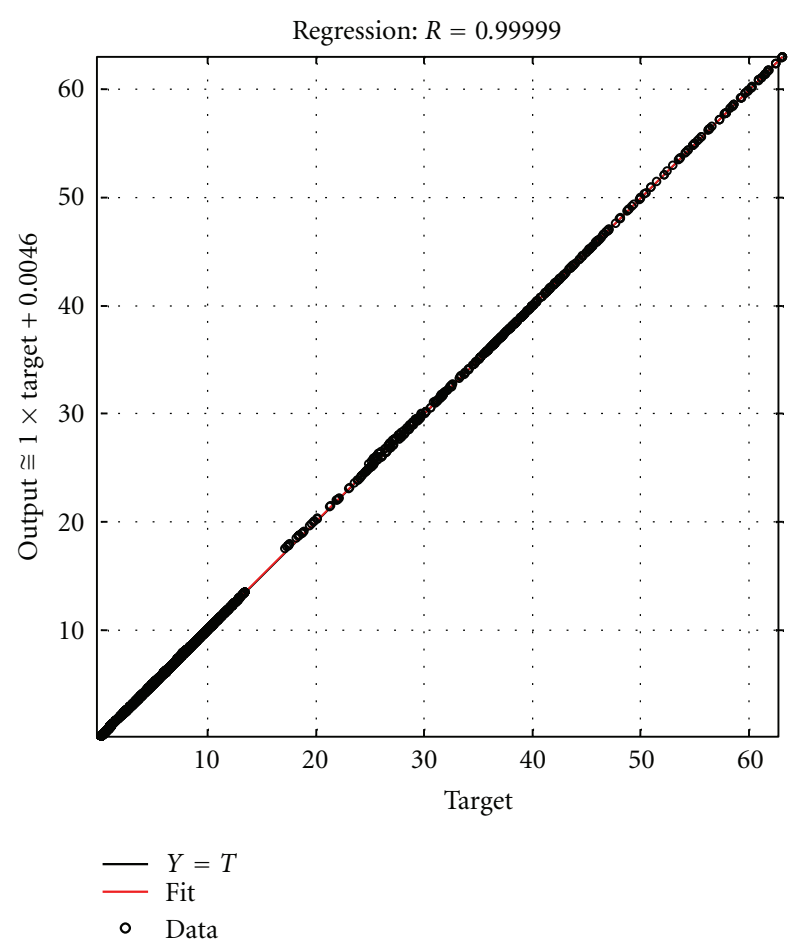

FIGURE 4: Regression analysis between the ANN output and the corresponding target for real power allocation.

4.1. Training. After the input and target for training data is created, it can be made more efficient by scaling the network inputs and targets so that they always fall within a specified range. In this case the minimum and maximum value of input and output vectors is used to scale them in the range of -1 and +1 . The next step is to divide the data $(D$ and $T)$ up into training. In this case, 100 samples $(60 \%)$ of data are used for the training.

The training of the RBF ANN consists of two separate stages. The first step is to find the centers parameter by using the $k$-means clustering algorithm. After a number of trials, $k$ is taken as 14 and the $\beta$ as 17 . These values give reasonable accuracy during training. In the second training stage, the second layer weights in connections between the hidden layer and the output layer are determined using the least squares based on minimization of quadratic errors of RBF ANN network output values over the set of training input-output vector pairs. The training performance is shown in Figure 3. From Figure 3, it can also be seen that the training goal is achieved in 2 epochs with performance equal to $3.13 E-6$. The training time taken by the RBF ANN is $232 \mathrm{msec}$ using an Intel Core $2 \mathrm{Duo}, 2 \mathrm{GHz}$ computer.

4.2. Pretesting and Simulation. After the networks have been trained, the next step is to simulate the network. The entire training data is used in pretesting. After simulation, the obtained result from the trained network is evaluated with a linear regression analysis. In real power allocation scheme, the regression analysis for the trained network is shown in Figure 4 . The correlation coefficient, $(R)$, in this case is very 


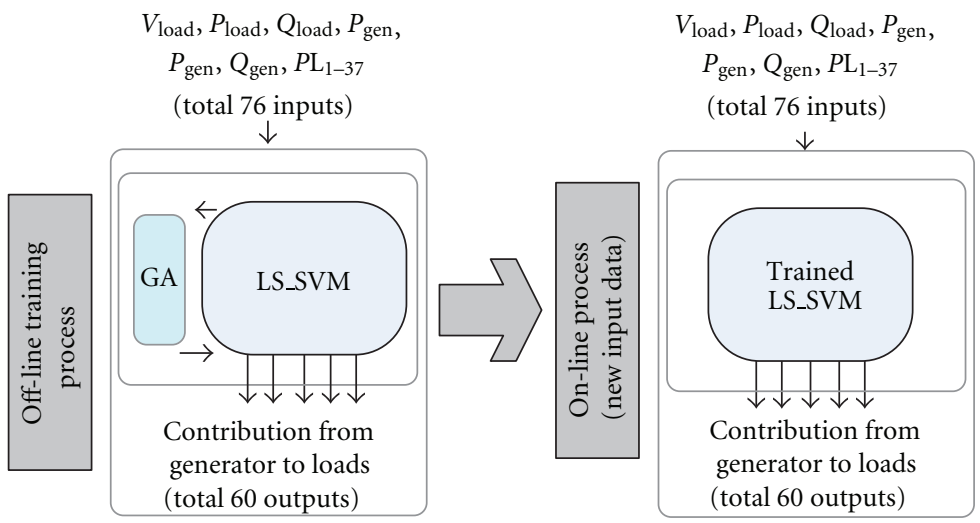

FIGURE 5: Description of inputs and outputs of the training and simulation data for GA-LSSVM for real power transfer allocation.

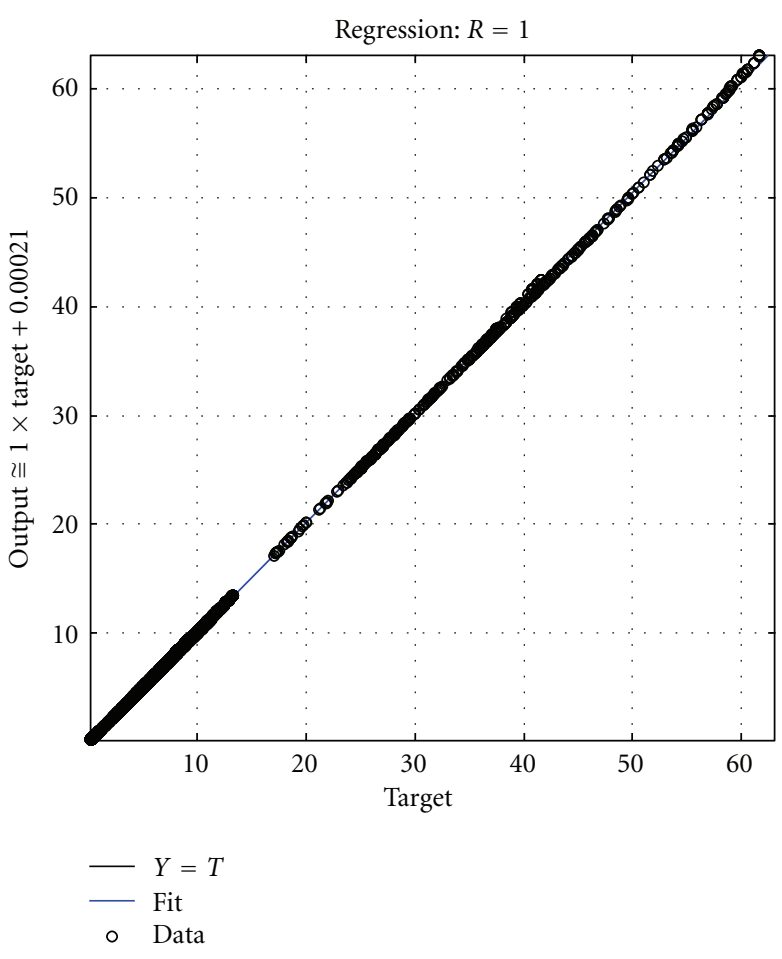

FIGURE 6: Regression analysis between the GA-LSSVM output and the corresponding target for real power allocation.

close to the one which indicates perfect correlation between the proposed method and the output of the neural network.

\section{GA-LSSVM Design for Real Power Allocation}

In order to find the optimal value of LS-SVM parameters, namely regularization parameter, $\gamma$ and Kernel RBF parameter, $\sigma^{2}$, the continuous GA is used. The properties of GA to find the optimal $\gamma$ and $\sigma^{2}$ are set as shown in Table 1 .

The GA-LSSVM design for real power allocation is further elaborated for the same 25 bus system shown in Figure 1. Here again the same input and target data sets used in the previous AI power transfer allocation are used to
TABle 1: Properties of GA to find the optimal $\gamma$ and $\sigma^{2}$.

\begin{tabular}{lc}
\hline Property & Type and values \\
\hline Selection & Roulette wheel \\
Crossover & Modified single point arithmetic crossover \\
Crossover probability & 0.9 \\
Mutation probability & 0.1 \\
Population & 40 \\
Maximum iteration & 20 \\
\hline
\end{tabular}

avoid discrimination among all methods considered in this paper. Input data $(D)$ for developed GA-LSSVM contains 76 variables and target $(T)$ contains 60 output parameters which is exactly the same as that used in RBF ANN method, Figure 5 summarize the description of inputs and outputs of the training and simulation stages for GA-LSSVM for real power allocation.

5.1. Training. After the input and target of training data have been created, the next step is to divide the data ( $D$ and $T$ ) up into training, validation, and testing subsets. Here again, 100 samples of data are used for the training out of 168 hour samples collected for training process.

The property of regularization parameter, $\gamma$, and Kernel RBF, $\sigma^{2}$ are decided through the hybrid GA-LSSVM model that has been discussed above. From the testing phase of GALSSVM model, the final value of $\gamma$ is set to 9923.9 and $\sigma^{2}$ is set to 1347.8 . It took $210.52 \mathrm{sec}$ to optimize the $\gamma$ and $\sigma^{2}$ values using the same computer. These LSSVM parameters yield a reasonably accurate output of the predictive model that has been designed. The mean square error (MSE) at pre-testing stage is $2.238 E-5$ which show that the estimation by GALSSVM model and the training data are having the similar characteristics.

5.2. Pretesting and Simulation. After the hybrid GA-LSSVM model has been trained, the entire 168 samples of data are used in pretesting. After simulation, the obtained result from the trained model is evaluated with the linear regression analysis as shown in Figure 6. The correlation coefficient, $(R)$, 


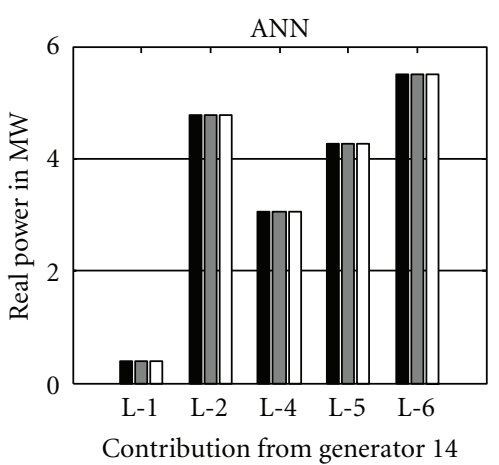

(a)

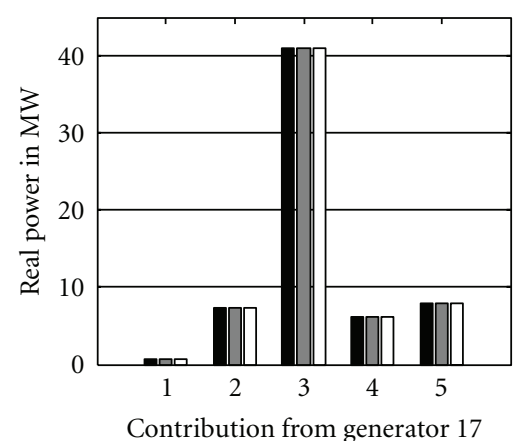

(d)

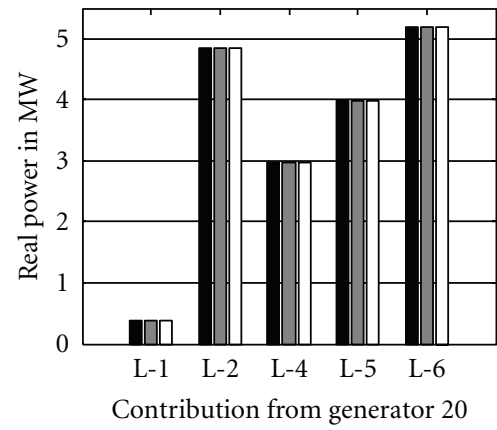

(g)
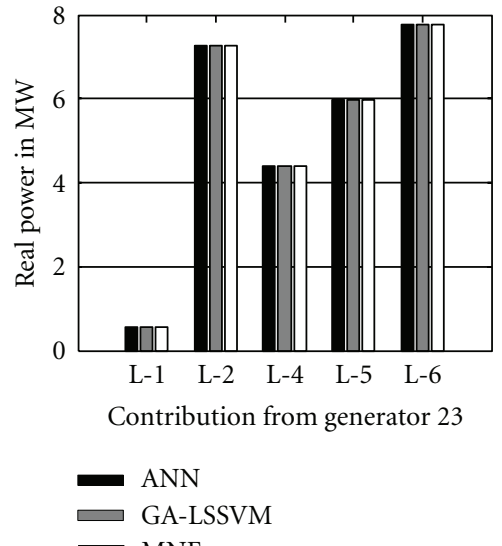

$\square$ GA-LSSVM

$\square$ MNE

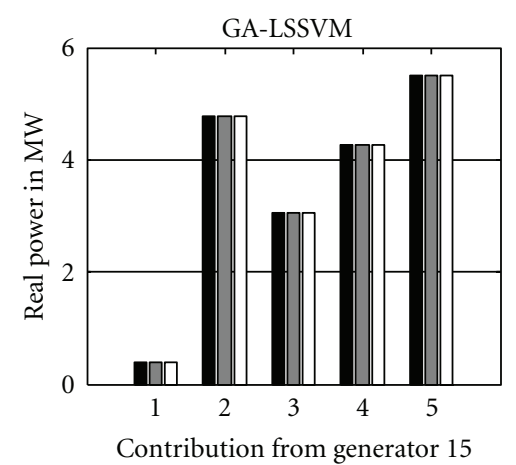

(b)

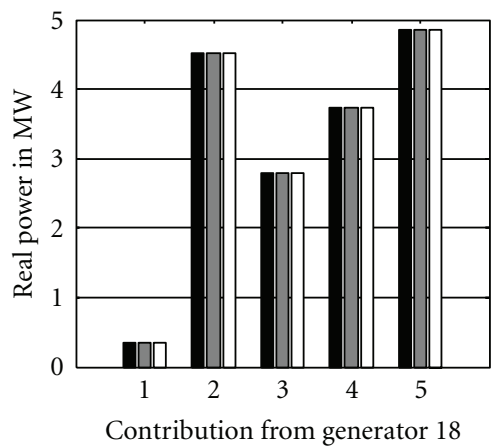

(e)

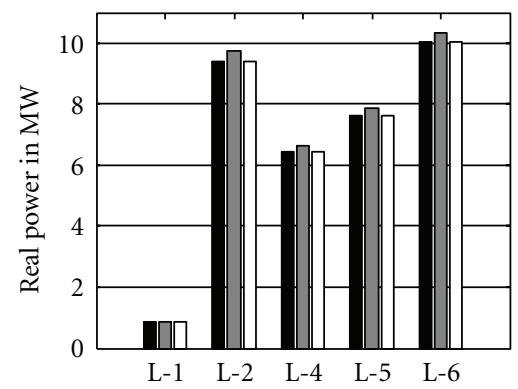

Contribution from generator 21

(h)
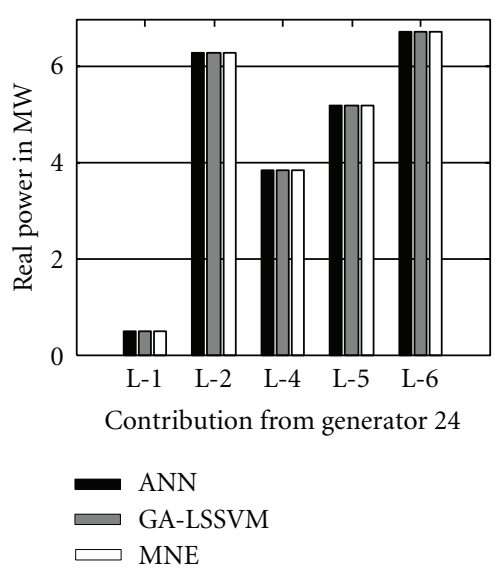

(k)

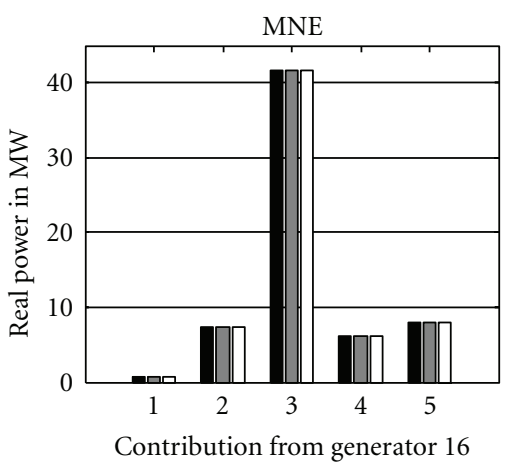

(c)

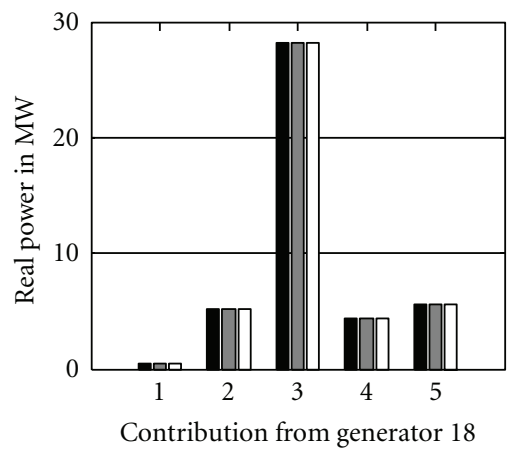

(f)

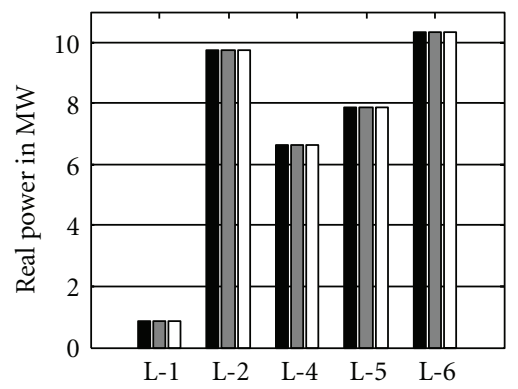

Contribution from generator 22

(i)

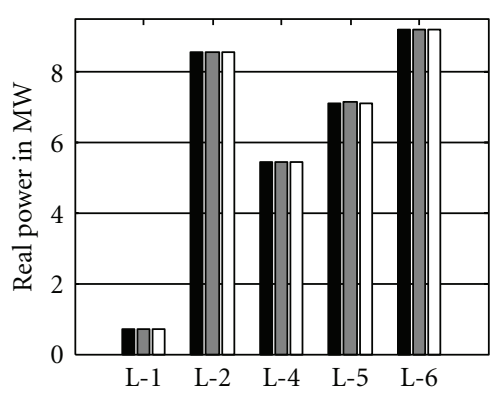

Contribution from generator 25

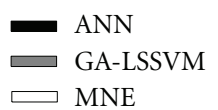

(1)

Figure 7: Analysis of real power allocation for the 25-bus equivalent system during hour 33. 

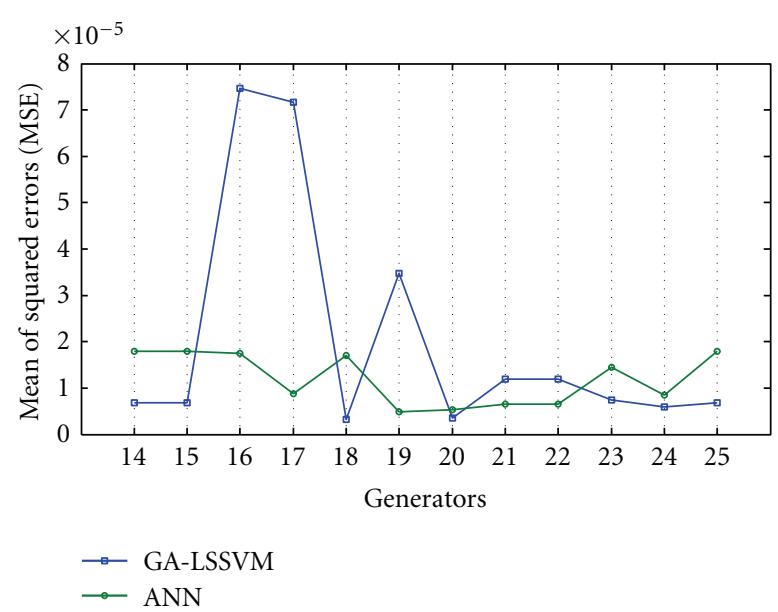

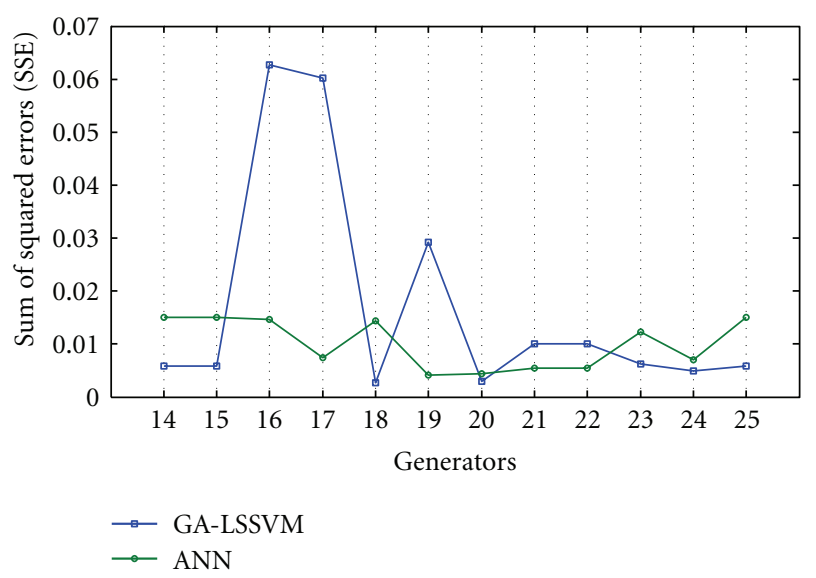

(b)

FIGURE 8: Quantitative performance of various AI methods for untrained data, (a) MSE errors in power transfer allocation of individual generators (b) SSE errors in power transfer allocation of individual generators.

in this case is equal to 1 which indicates a good correlation between MNE method and GA-LSSVM.

\section{Results and Analysis}

A number of simulations have been carried out to exhibit the accuracy of the developed AI power transfer allocation methods with the same 25-bus equivalent system of south Malaysia. The scenario is a decrement by $5 \%$ of the real and reactive load demand from the nominal trained pattern for 1 week (168 hours). Besides it also assumed that all generators also decrease their production proportionally according to this variation in the load demands. This assumption is being made to ensure that all real power generation of generator at buses 14 to 25 varies in respond to the varying daily load pattern of the loads. The allocation of real power to loads using proposed AI methods on hours 33 out of 168 hours is presented in Figure 7 along with the result obtained through MNE method. From Figure 7, it can be noted that the result obtained by the AI methods output in this paper is well comparable with the result of MNE method. The difference of real power between generators in both AI methods and MNE method is very small during this hour which is less than or equal to $0.400 \mathrm{~kW}$. The consumer located at bus 4 consumed the highest demand compared to other consumers in this hour. Consequently, the contribution of real power due to generators 16,17 , and 19 located at the same bus provides more real power to load at bus 4 by all AI methods and MNE method as well. This result also justifies the physical meaning of MNE method as these generators are the nearest to load at bus 4 .

To further evaluate the quantitative performance, mean square error (MSE) and sum of square error (SSE) observed by individual generator allocations and overall MSE and SSE encountered by each AI method are obtained. Figure 8 shows the MSE and SSE values introduced by each AI method they are subjected to untrained data. It can be observed that
MSE and SSE errors for both GA-LSSVM method and ANN method are comparable. In addition, it can also be noted that error differences between generator allocations in case ANN method is minimum which ranges between $1.71 E-05$ and 4.86E-06 for MSE error and 0.0147 and 0.0041 for SSE error.

The overall comparison of two AI methods that is used in power transfer allocation is exhibited in Table 2. It can be noted that mathematical model type (MNE) takes much longer time for training compared with multioutput model types like ANN and SVM. When comparing with overall MSE and SSE errors encountered during data simulation, the best performance is provided by ANN method whose MSE and SSE are found to be $1.19 E-5$ and 0.1203 , respectively. All in all, it can be concluded that ANN method is the best to use for power transfer allocation because it takes very short training time in model development and provides more accurate results in less simulation time as shown in Table 2.

\section{Conclusion}

This paper has presented two preference AI methods that can be used to identify the real power transfer between generators and load. The developed AI method adopts real power allocation outputs determined by MNE technique as the trainer during the model development phase. The robustness of the two AI methods has been demonstrated on the 25-bus equivalent system of south Malaysia. From the results, the following conclusions can be attained.

(1) The AI power transfer allocation methods provide the results in a faster and convenient manner.

(2) Among two AI methods, ANN method provides the most accurate results while GA-LSSVM method also gives an acceptable result.

(3) In terms of training, ANN and GA-LSSVM require comparably small training time. 
TABLE 2: Qualitative and quantitative comparison of two AI power transfer allocation methods.

\begin{tabular}{lccccc}
\hline Method & Model type & $\begin{array}{c}\text { Training time } \\
(\mathrm{sec})\end{array}$ & $\begin{array}{c}\text { Simulation time } \\
(\mathrm{msec})\end{array}$ & Overall MSE error for new data & Overall SSE error for new data \\
\hline ANN & Multioutput & 0.2321 & 21.99 & $1.19 E-05$ & 0.1203 \\
GA-LSSVM & Multioutput & 0.2388 & 23.89 & $2.05 E-05$ & 0.2064 \\
MNE & Mathematical & - & 360.00 & - & - \\
\hline
\end{tabular}

(4) The ANN-based method is the most suitable to be adapted in true application of real power allocation.

(5) The proposed AI method can resolve some of the difficult real power pricing and costing issues to ensure fairness and transparency in the deregulated environment of power system operation.

\section{Acknowledgments}

The authors wish to acknowledge the Ministry of Higher Education, Malaysia (MOHE) for the financial funding of this project, and Universiti Teknologi Malaysia and Universiti Kabangsaan Malaysia, for providing infrastructure and moral support for the research work.

\section{References}

[1] H. Shareef and M. W. Mustafa, "Real and reactive power allocation in a competitive market," WSEAS Transactions on Power Systems, vol. 1, pp. 1088-1094, 2006.

[2] R. Reta and A. Vargas, "Electricity tracing and loss allocation methods based on electric concepts," IEE Proceedings: Generation, Transmission and Distribution, vol. 148 , no. 6, pp. 518 522, 2001.

[3] Y. C. Chang and C. N. Lu, "Electricity tracing method with application to power loss allocation," International Journal of Electrical Power and Energy System, vol. 23, no. 1, pp. 13-17, 2001.

[4] A. Zobian and M. D. Ilić, "Unbundling of transmission and ancillary services Part I: technical issues," IEEE Transactions on Power Systems, vol. 12, no. 2, pp. 539-548, 1997.

[5] J. H. Teng, "Power flow and loss allocation for deregulated transmission systems," International Journal of Electrical Power and Energy Systems, vol. 27, no. 4, pp. 327-333, 2005.

[6] H. Shareef, M. W. Mustafa, S. Abd Khalid, A. Khairuddin, A. Kalam, and A. Maung Than Oo, "Real and reactive power transfer allocation utilizing modified Nodal equations," International Journal of Emerging Electric Power Systems, vol. 9, no. 6, article 4, 2008.

[7] J. Bialek, "Tracing the flow of electricity," IEE Proceedings Generation Transmission \& Distribution, vol. 143, no. 4, pp. 313-320, 1996.

[8] F. F. Wu, "Power transfer allocation for open access using graph theory-fundamentals and applications in systems without loopflow," IEEE Transactions on Power Systems, vol. 15, no. 3, pp. 923-929, 2000.

[9] M. W. Mustafa, H. Shareef, and M. R. Ahmad, "An improved usage allocation method for deregulated transmission systems," in Proceedings of the 7th International Power Engineering Conference (IPEC '05), pp. 406-411, December 2005.

[10] S. Abdelkader, "Efficient computation algorithm for calculating load contributions to line flows and losses," IEE
Proceedings: Generation, Transmission and Distribution, vol. 153, no. 4, pp. 391-398, 2006.

[11] D. K. Ron and A. G. Strbac, "Contributions of individual generators to loads and flows," IEEE Transactions on Power Systems, vol. 12, no. 1, pp. 52-60, 1997.

[12] M. W. Mustafa, S. N. Khalid, H. Shareef, and A. Khairuddin, "Reactive power transfer allocation method with the application of artificial neural network," IET Generation, Transmission and Distribution, vol. 2, no. 3, pp. 402-413, 2008.

[13] N. B. Dev Choudhury and S. K. Goswami, "Artificial intelligence solution to transmission loss allocation problem," Expert Systems with Applications, vol. 38, no. 4, pp. 3757-3764, 2011.

[14] A. R. Abhyankar, S. A. Soman, and S. A. Khaparde, "Optimization approach to real power tracing: an application to transmission fixed cost allocation," IEEE Transactions on Power Systems, vol. 21, no. 3, pp. 1350-1361, 2006.

[15] M. H. Sulaiman, M. W. Mustafa, and O. Aliman, "Transmission loss and load flow allocations via genetic algorithm technique," in Proceedings of the IEEE Region 10 Conference (TENCON '09), pp. 1-5, November 2009.

[16] H. Shareef, A. Mohamed, S. N. Khalid, M. W. Mustafa, and A. Khairuddin, "Real power transfer allocation utilizing support vector machine," in Proceedings of the International Conference of Electrical Energy and Industrial Electronic Systems (EEIES '09), pp. 1-7, Penang, Malaysia, December 2009.

[17] J. A. K. Suykens and J. Vandewalle, "Least squares support vector machine classifiers," Neural Processing Letters, vol. 9, no. 3, pp. 293-300, 1999.

[18] J. Nagi, K. S. Yap, S. K. Tiong, S. K. Ahmed, and A. M. Mohammad, "Detection of abnormalities and electricity theft using genetic support vector machines," in Proceedings of the IEEE Region 10 Conference (TENCON'08), pp. 1-6, November 2008.

[19] M. W. Mustafa, M. H. Sulaiman, H. Shareef, and S. N. Abd. Khalid, "Reactive power tracing in pool-based power system utilising the hybrid genetic algorithm and least squares support vector machine," IET Generation, Transmission and Distribution, vol. 6, no. 2, pp. 133-141, 2012.

[20] D. S. Broomhead and D. Lowe, "Multivariable functional interpolation and adaptive networks," Complex System, vol. 2, pp. 321-355, 1988.

[21] F. Girosi and T. Poggio, "Networks and the best approximation property," Biological Cybernetics, vol. 63, no. 3, pp. 169-176, 1990.

[22] V. N. Vapnik, The Nature of Statistical Learning Theory, New York, NY, USA, 2nd edition, 1995.

[23] J. A. K. Suykens, T. V. Gestel, J. De Brabanter, B. De Moor, and J. Vandewelle, Least Squares Support Vector Machines, World Scientific, Singapore, 2002.

[24] M. Espinoza, J. A. K. Suykens, and B. De Moor, "Fixed-size least squares support vector machines: a large scale application in electrical load forecasting," Computational Management Science, vol. 3, no. 2, pp. 113-129, 2006. 

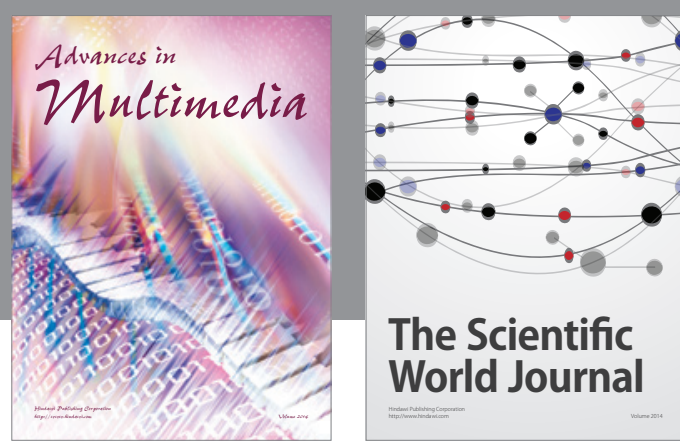

The Scientific World Journal
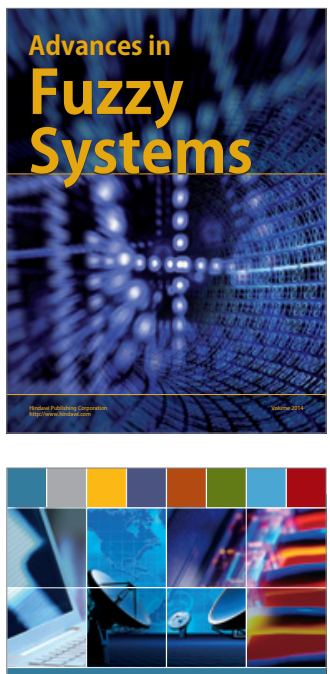

Computer Networks and Communications
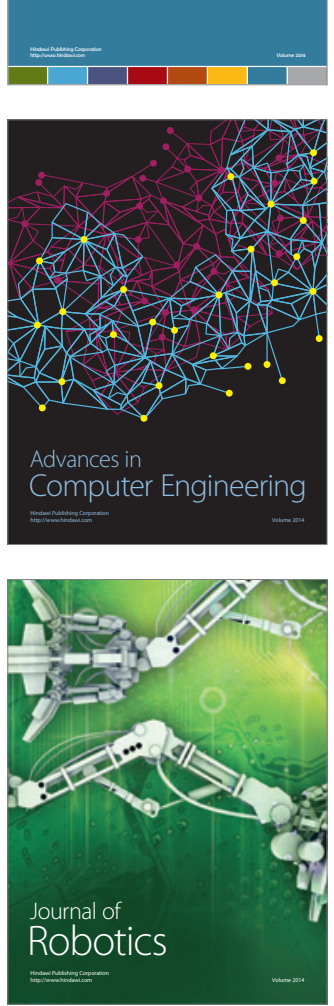
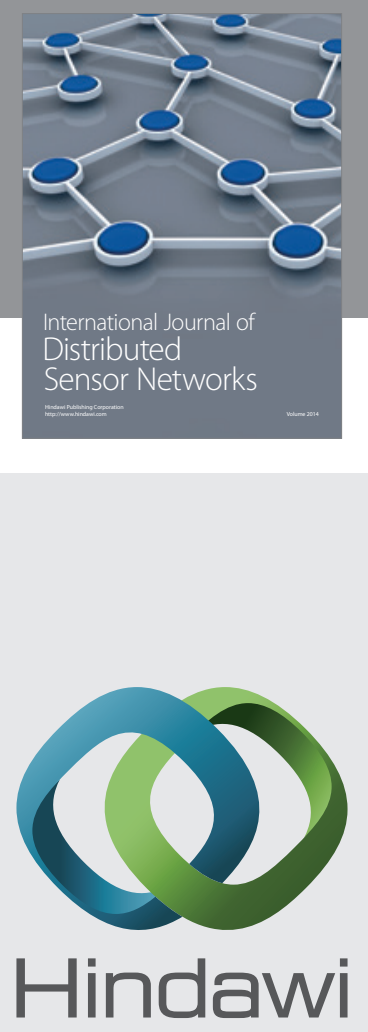

Submit your manuscripts at

http://www.hindawi.com
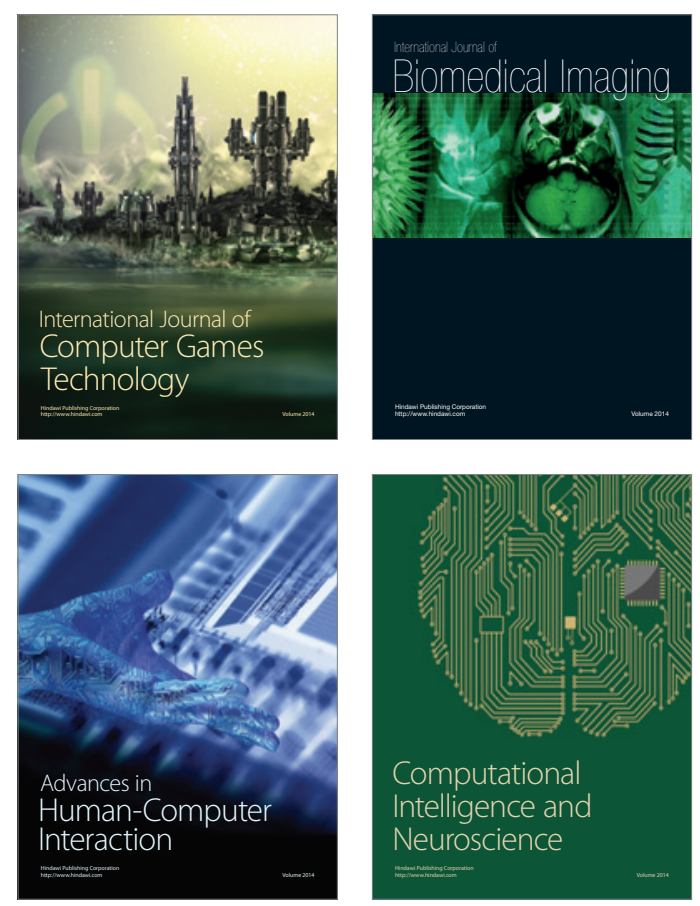
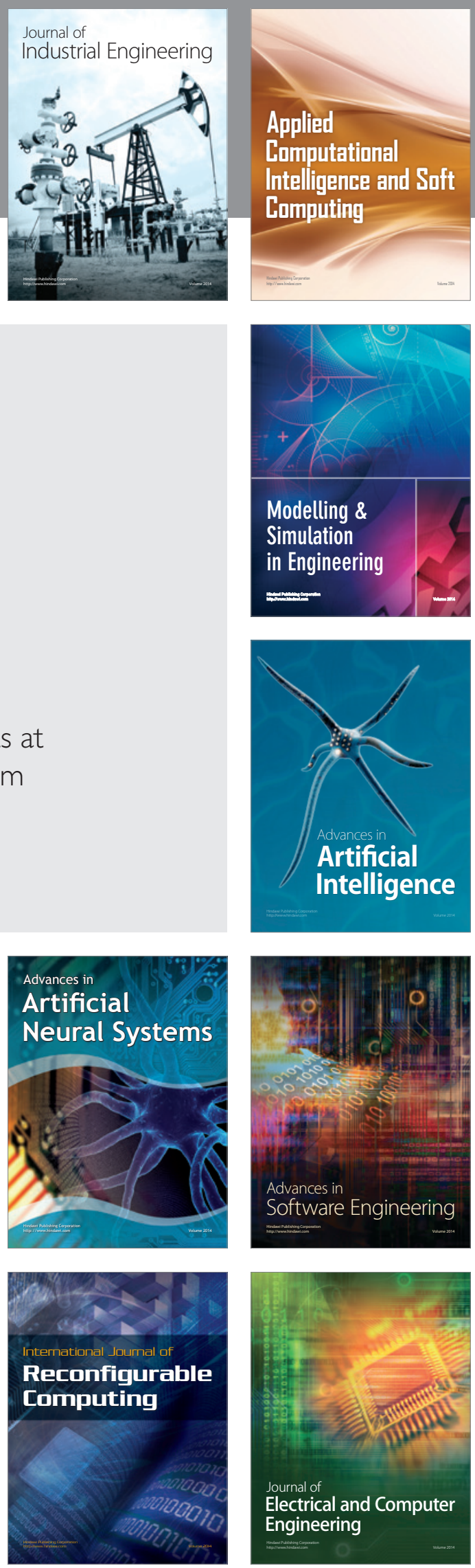